تأثير استخدام الأحواض الانتقائية اللاهوائية على كفاعة أنظمة الحماة المنشطة المستمرة الجريان

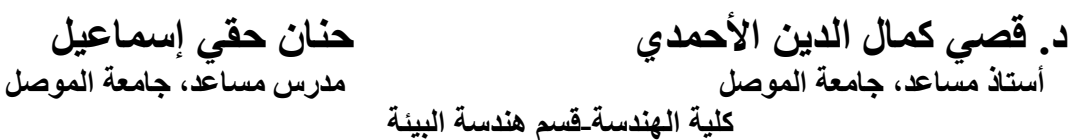

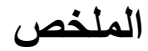

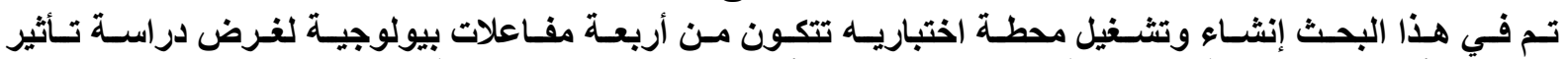

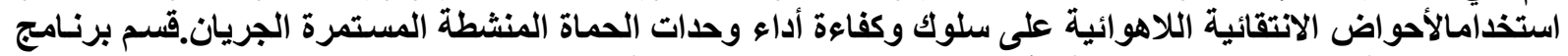

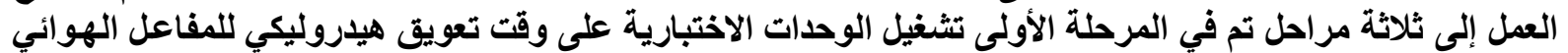

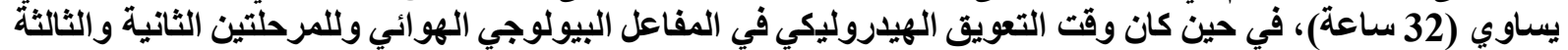

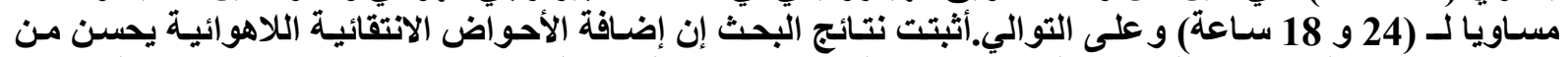

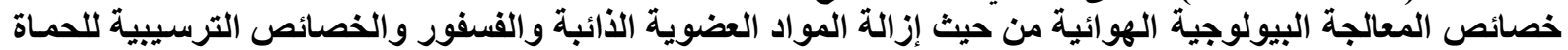

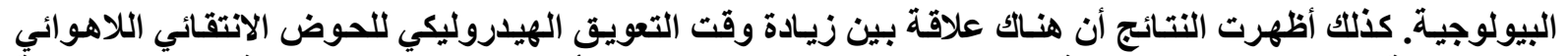

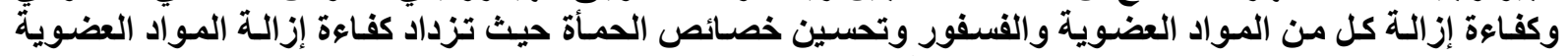

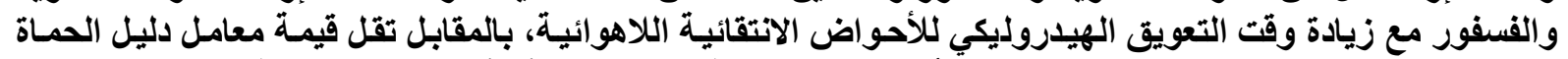

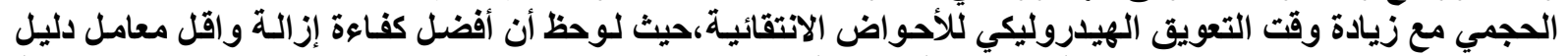

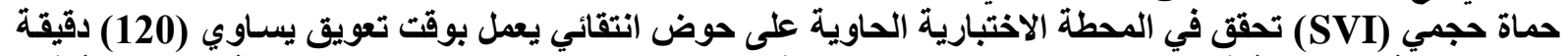

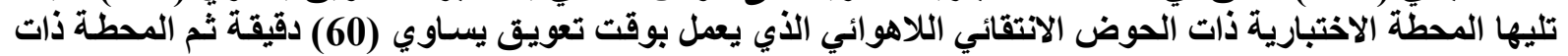
الحوض الانتقائي الذي يعمل بوقت تعويق يسأوي (30) دقيقة.

\title{
Effect of Using Anaerobic Selectors on the Performance of Continuous Flow Activated Sludge Systems
}

\section{Dr. Kossay K. Al-Ahmady}

Ass. Professor, University of Mosul,

\section{HananHaqeIsmaeel}

Ass. Lecturer; University of Mosul,

College of Engineering, Environmental Eng. Dept.

\section{Abstract}

In this research, four experimental plants have been constructed and operated in order to study the impact of using the anaerobic selectors on the behavior and efficiency of the extended aeration activated sludge system. The study program was divided into three stages. In the first stage, the experimental plants were run on the hydraulic retention time of the aerobic reactor equals to (32) hours, whereas, the hydraulic retention times in the biological aerobic reactors for the second and the third stages were (24 and 18 ) hours respectively. The results of the study revealed that; adding the anaerobic selectors improves the properties of the aerobic biological treatment in terms of removing soluble organic materials (CODs) and the efficiency of removing phosphor $\left(\mathrm{PO}_{4}\right)$. The results also showed that,the organic and phosphorus removal efficiency increases with increasing the hydraulic retention time of the anaerobic selector. In contrast the values of the sludge volume index (SVI) decreases with this increasing. Higher removal efficiencies were recorded in the experimental stations that include anaerobic selector, which operates on a hydraulic retention time of (120 min) followed by the plant with the selector of $(60 \mathrm{~min})$ detention time and in the last place the plant that include selector, which operates on a hydraulic retention time of $(30 \mathrm{~min})$. Keywords:Anaerobic selector, Activated sludge, HDT,SVI, Phosphorus removal, Organic removal 


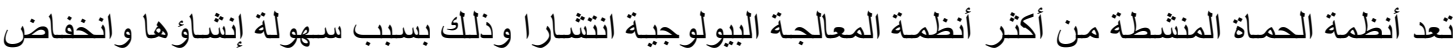

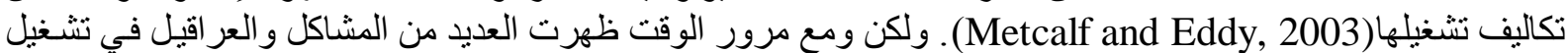

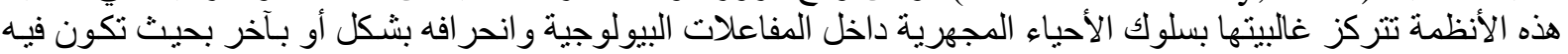

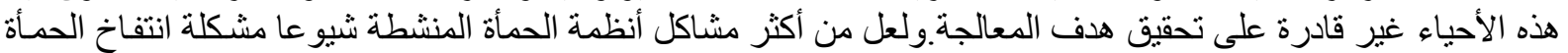

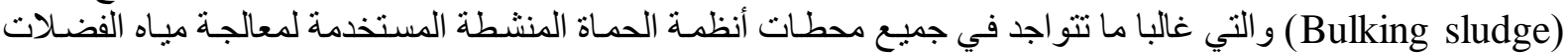

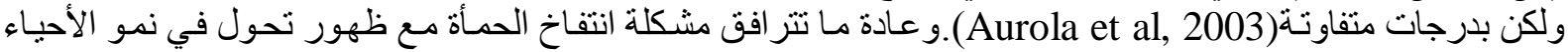

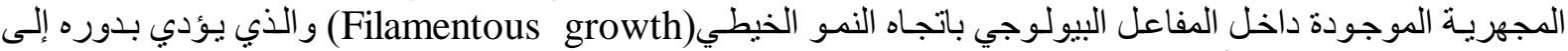

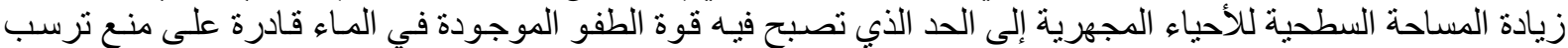

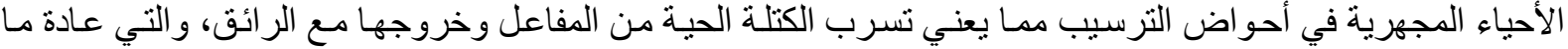

تتر افق في الحالات الثديدة مع فشل و انهيار المنظومة البيولوجية بشكل كامل (Metcalf and Eddy, 2003).

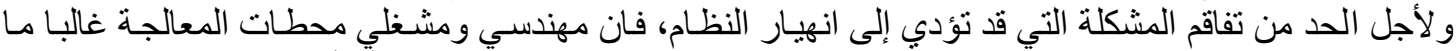

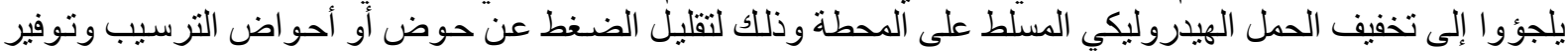

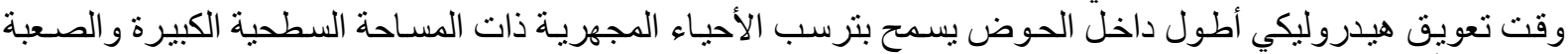

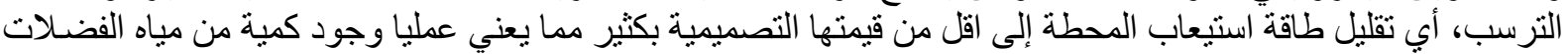
ستكون خارج قابلية المحطة.

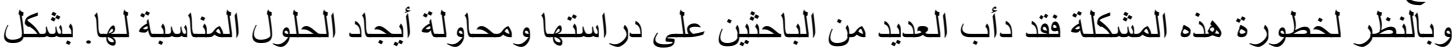

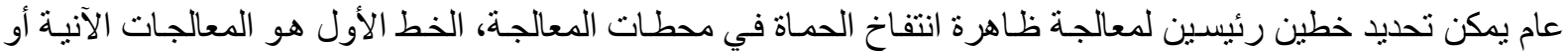

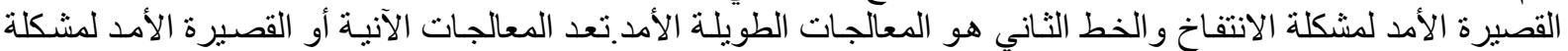

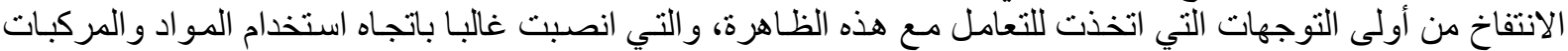

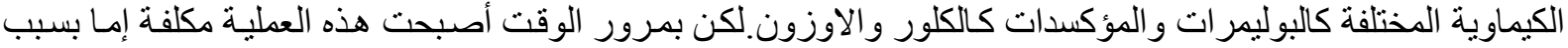

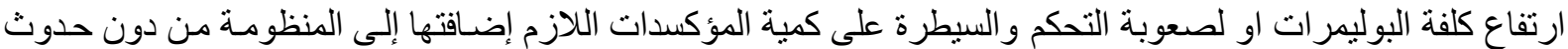

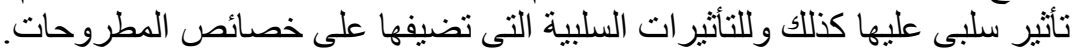

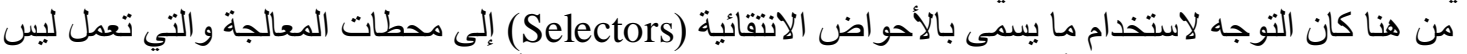

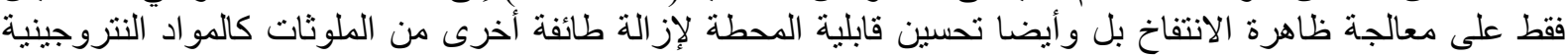

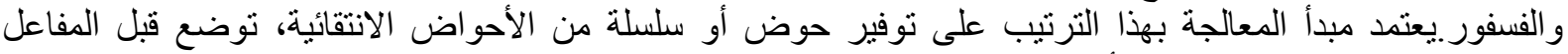

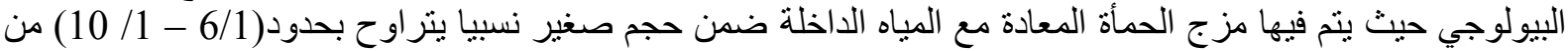

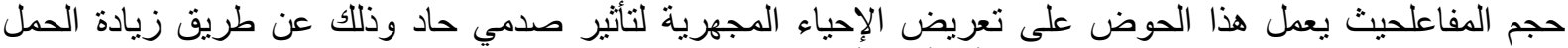

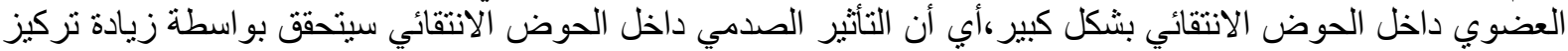

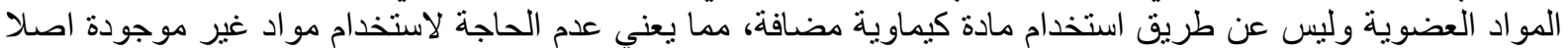
بمياه الفضلات، كذلك التخلص من التأثير السمومي المترتب عن هذا الاستخدام هذا إضافة لتقليل الكلف التشغيلية للمحطة

إن تعريض المنظومـة البيولوجيـة لهذه الصدمة غالبـا مـا يؤدي إلى موت معظم الأحياء المجهريـة ذات المسـاحة

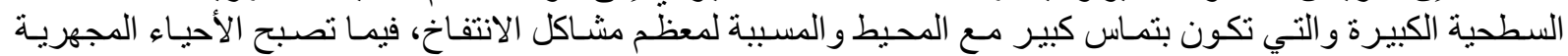

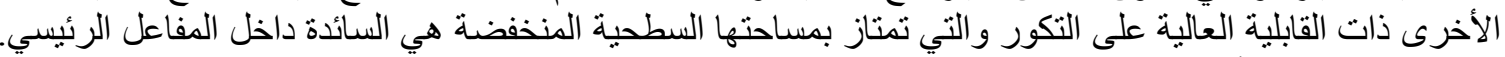

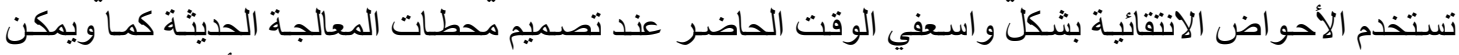

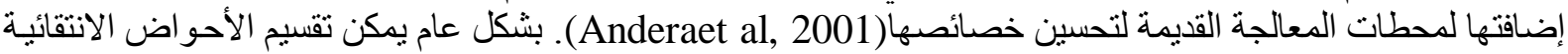

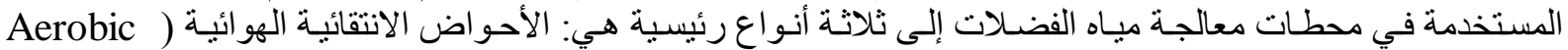

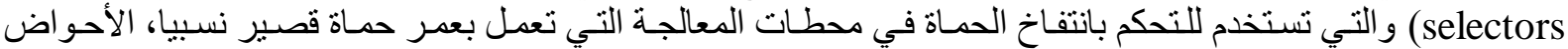

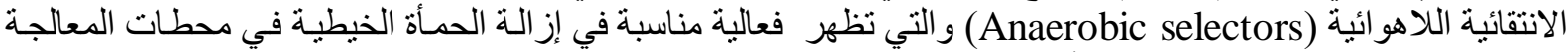

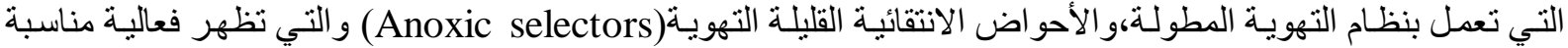

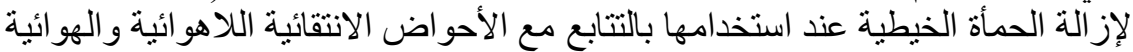
(Alberson and Hendricks, 1991)

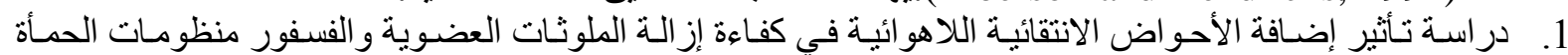

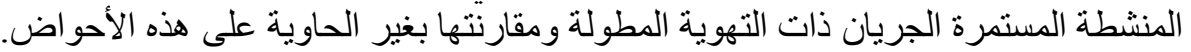


2. دراسة تأثير تغيير وقت التعويق الهيدروليكي في مفاعلات الحمأة المنشطة المستمرة الجريـان في كفاءة الإزالة عند وجود الأحو اض الائنقائية اللاهو ائية. 3. تأثير تغيير وقت التعويق الهيدروليكي في الأحو اض الانتقائية اللاهو ائية (Anaerobic selectors) و اثر ذلك في كفاءة الإز الة واستقر ارية النظام.

قام الباحث (Mangrum, 1998) بمعالجة مياه فضـلات مدنيـة باستخدام محطة تتألف من قسمين يعملالأول

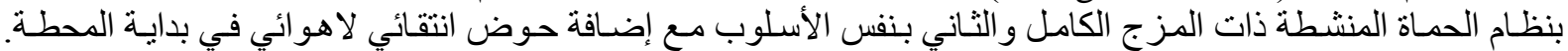

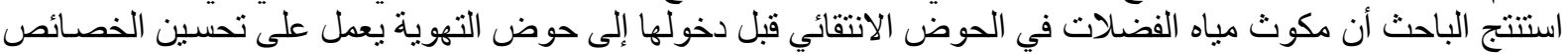

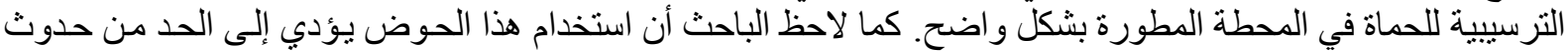

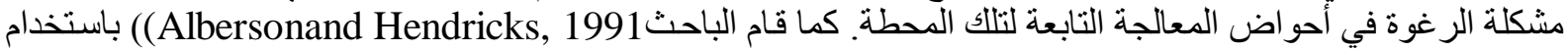

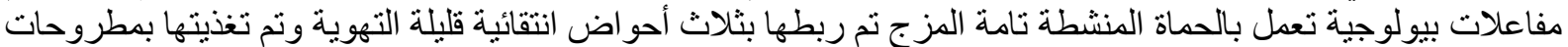

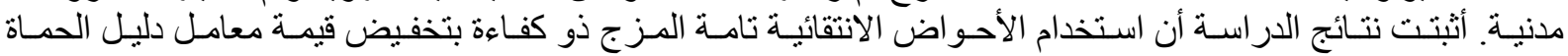

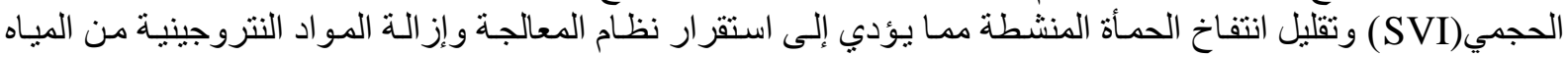

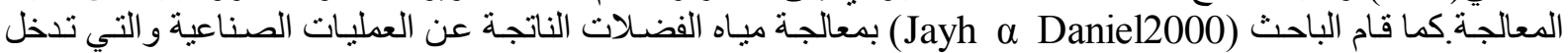

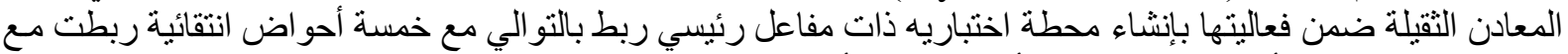

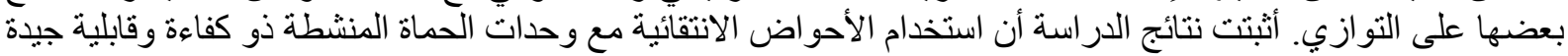

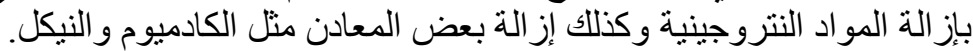

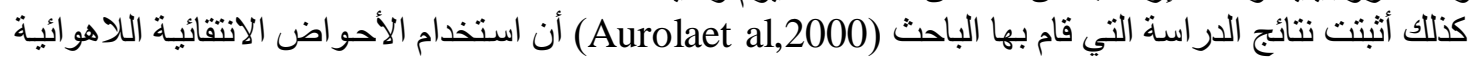

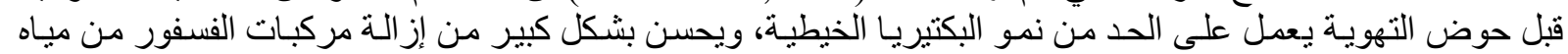

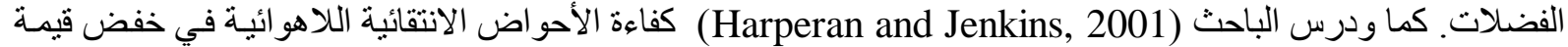

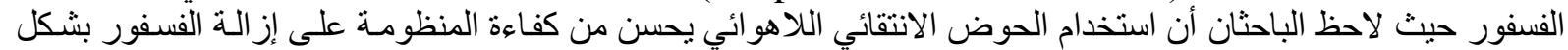

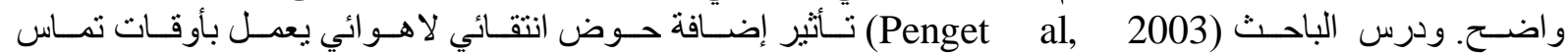

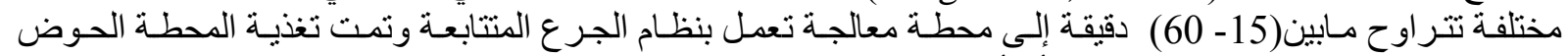

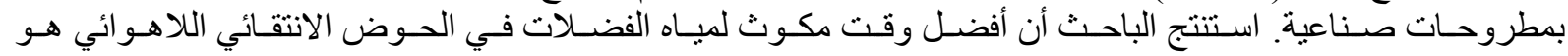

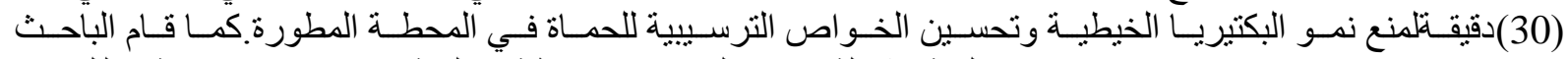

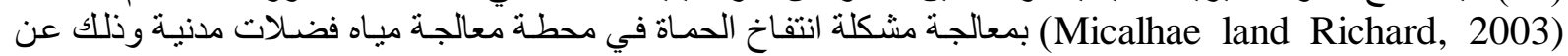

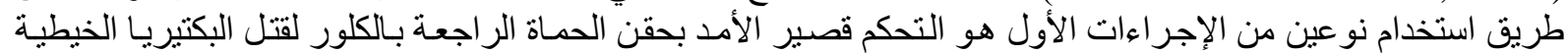

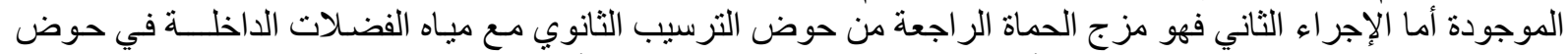

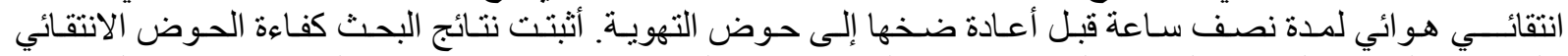

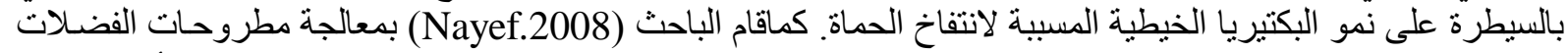

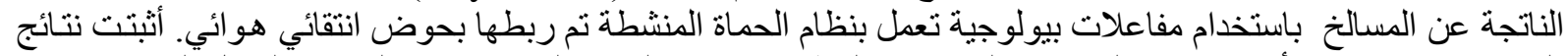

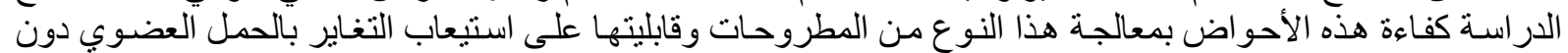
حصول فنل أو تذبذب بذه الاستقر ارية المعالجة.

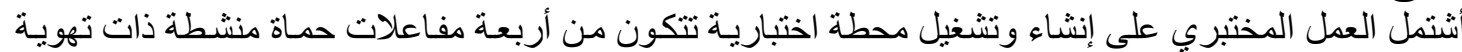

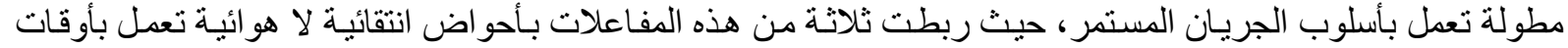

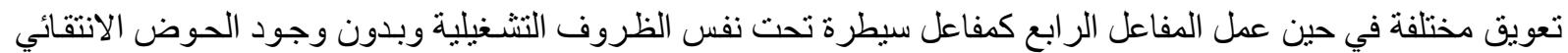

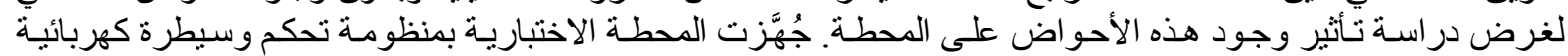

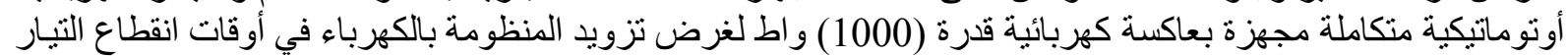

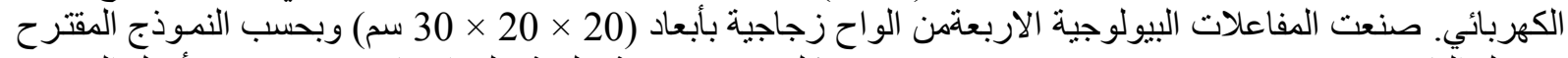

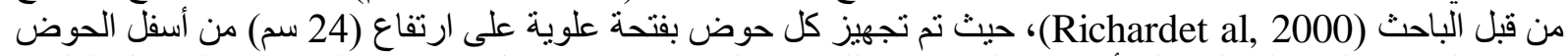

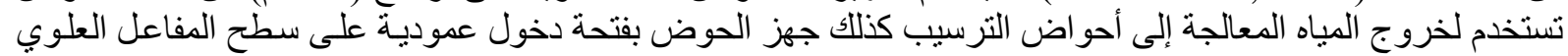

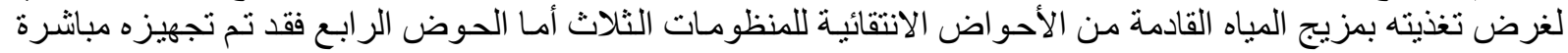
بمياه الفضلات من حوض التجهيز. يوضح المخططان (1) و (2) حوض التهوية وطريقة ربط المنظومة. 


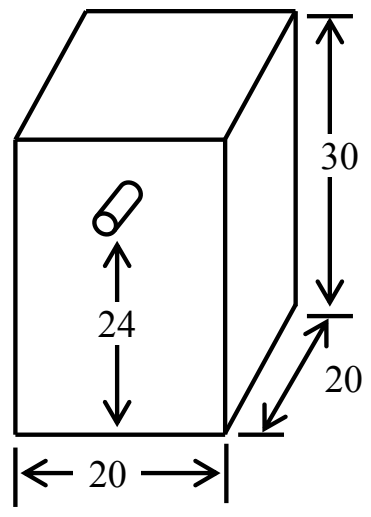

مخطط (1) المفاعل الحيوي الهوائي

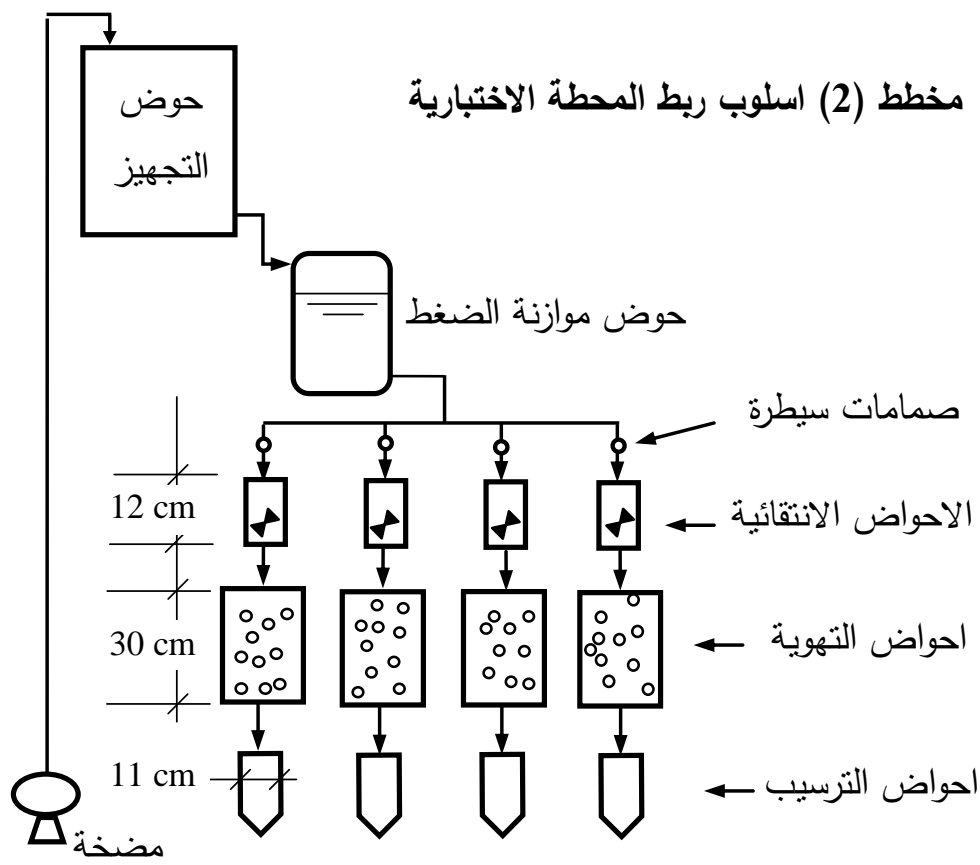

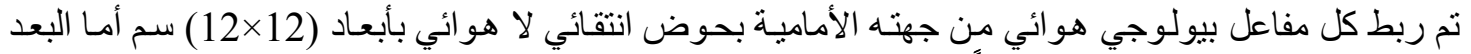

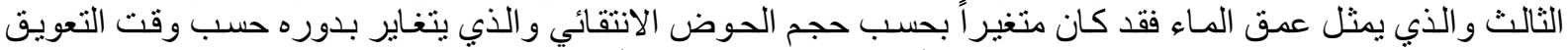

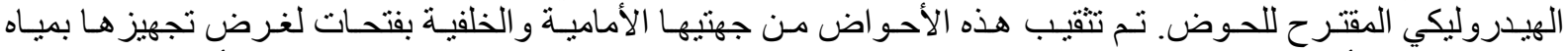

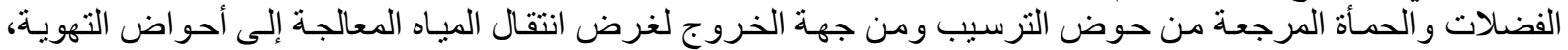

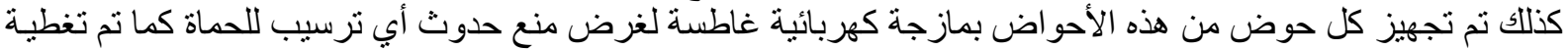

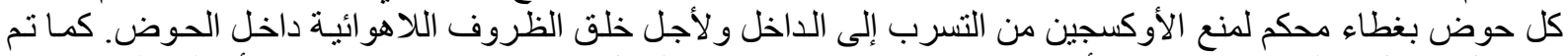

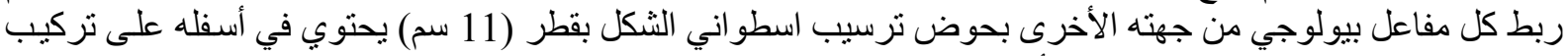

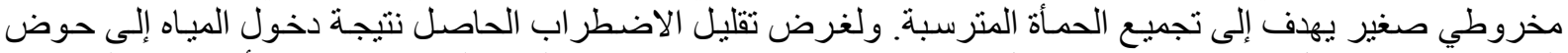

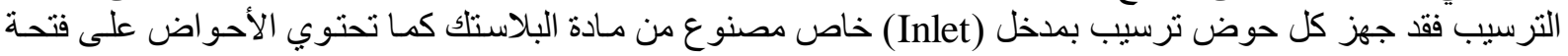

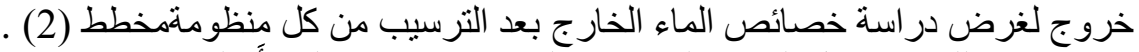

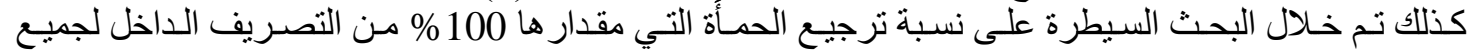

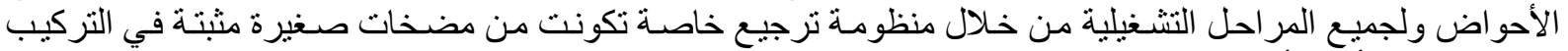

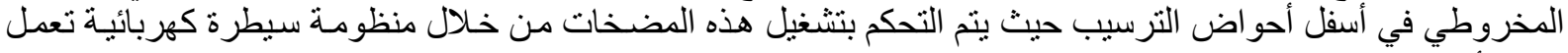

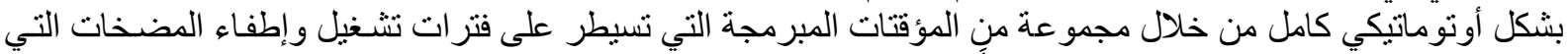

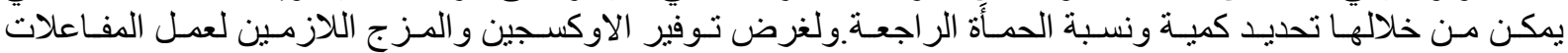

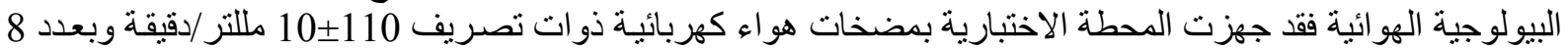

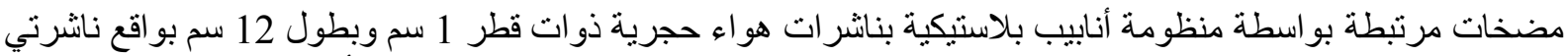

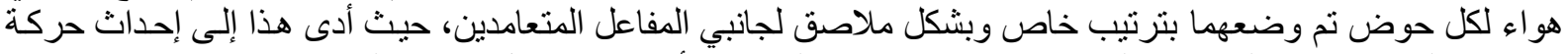

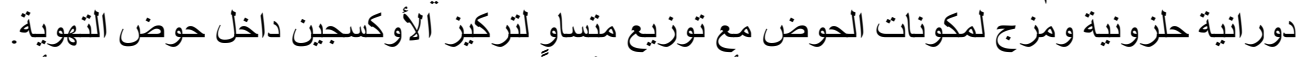

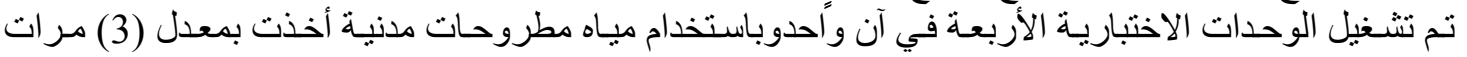

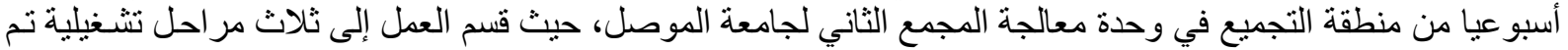

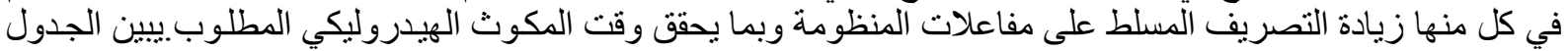

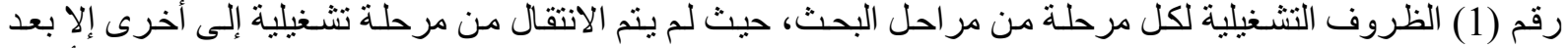

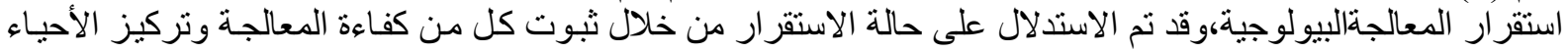

المجهرية فيها (Ramalho, 1977). 
النتائج والمناقشة الأنة

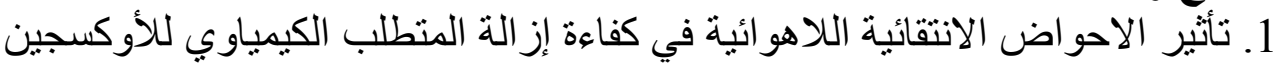

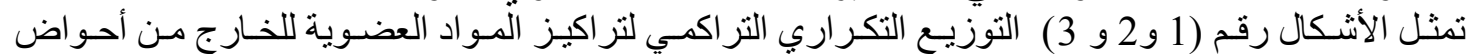

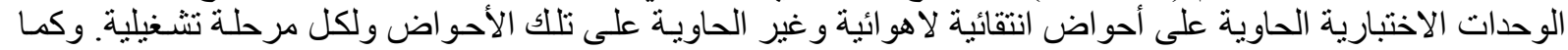

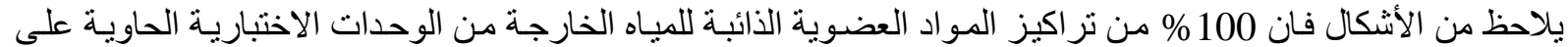

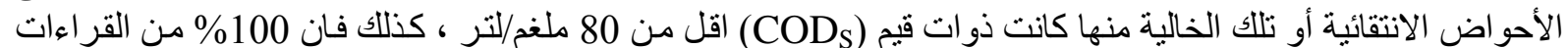

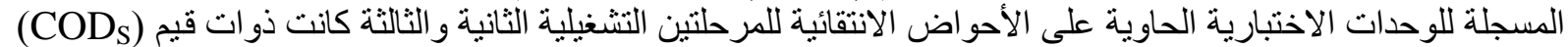

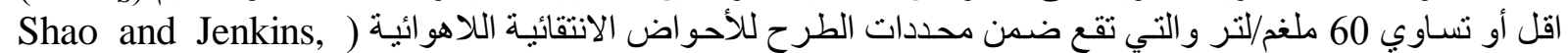

(1989

\begin{tabular}{|c|c|c|c|c|c|c|}
\hline \multicolumn{2}{|c|}{ الأحواض الانتقائية } & \multicolumn{2}{|c|}{ أحواض الترسيب } & \multicolumn{2}{|c|}{ أحواض التهوية } & \multirow[b]{2}{*}{ المرحلة } \\
\hline (ملتنز) & والهيدروليكي المكوث & (لترجم & وقتا الهيدروليكي & التصريف & وقلت المياروليكي & \\
\hline 150 & 30 & \multirow{3}{*}{1.2} & \multirow{3}{*}{4} & \multirow{3}{*}{7.2} & \multirow{3}{*}{32} & \multirow{3}{*}{ 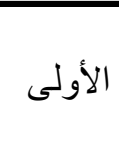 } \\
\hline 300 & 60 & & & & & \\
\hline 600 & 120 & & & & & \\
\hline 200 & 30 & \multirow{3}{*}{1.6} & \multirow{3}{*}{4} & \multirow{3}{*}{9.6} & \multirow{3}{*}{24} & \multirow{3}{*}{ الثانية } \\
\hline 400 & 60 & & & & & \\
\hline 800 & 120 & & & & & \\
\hline 250 & 30 & \multirow{3}{*}{2.1} & \multirow{3}{*}{4} & \multirow{3}{*}{12.8} & \multirow{3}{*}{18} & \multirow{3}{*}{ الثالثة } \\
\hline 500 & 60 & & & & & \\
\hline 1000 & 120 & & & & & \\
\hline
\end{tabular}

جدول (1) المراحل التثغئية الثثلاثة للوحدات الاختبارية
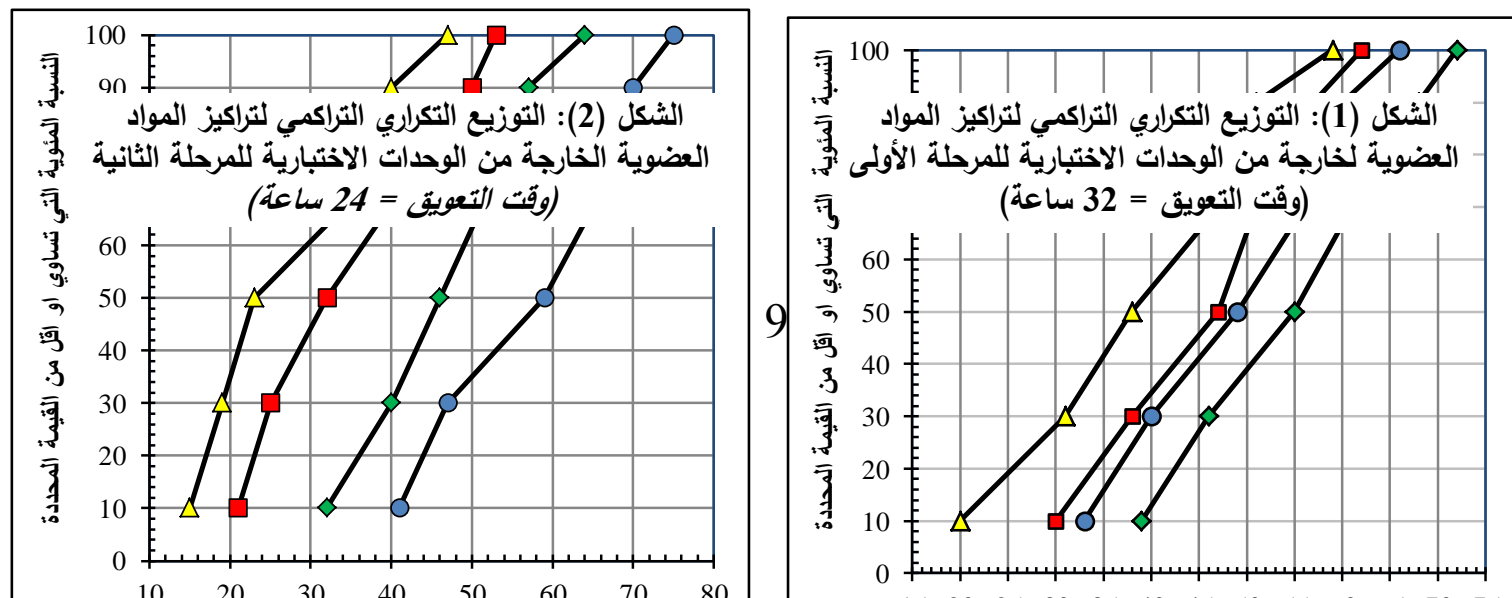


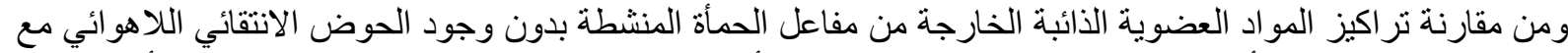

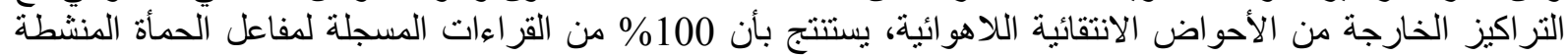

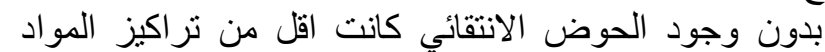

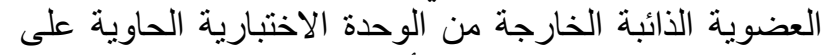

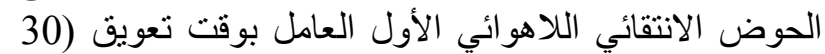

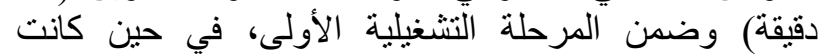

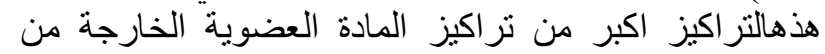

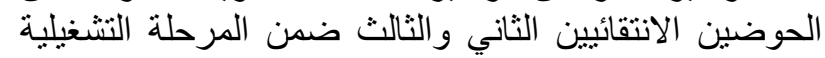

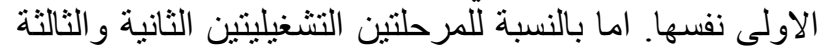

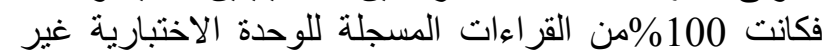

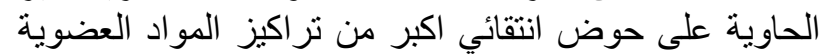

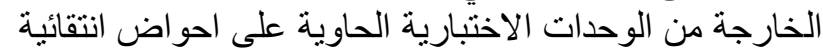

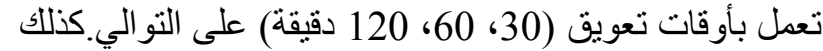

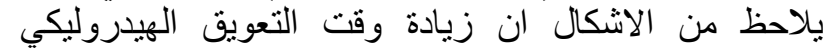

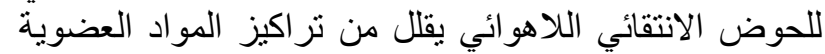

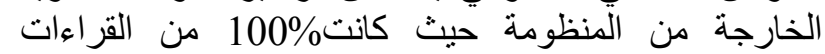

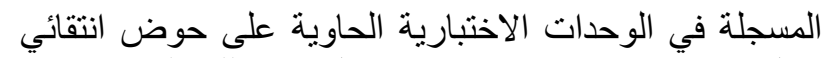

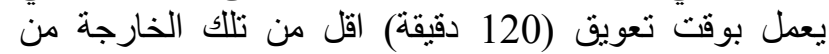

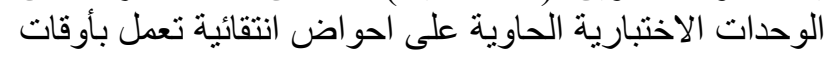

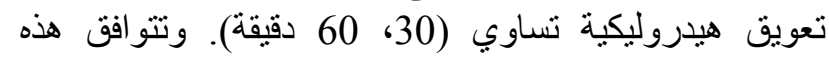
النتيجة مع ما ما اورده الباحثين (Tomlinson, 1978) (Richardet al, 2000)

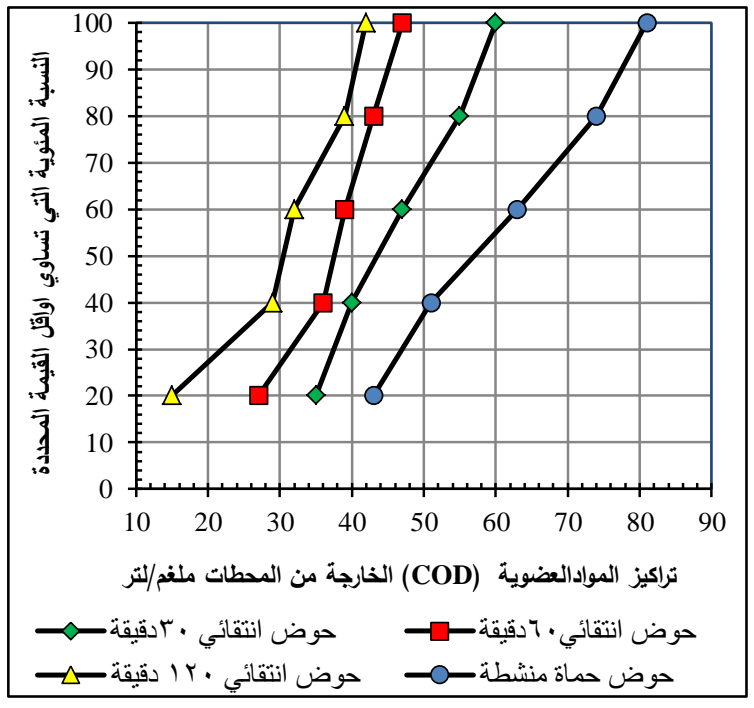

الثكل (3): التوزيع التكراري التراكمي لتراكيز المواد العضوية الخارجة من الوحدات الاختبارية للمرحلة التثغيلية الثالثة

(وقت التعويث = 18 ساعة)

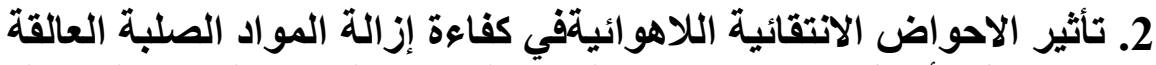

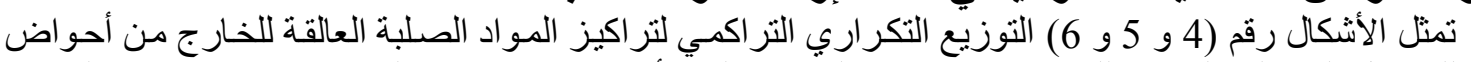

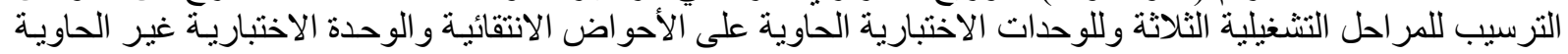
على حوض انتقائي اللمر احل التشغيلية الثناث.

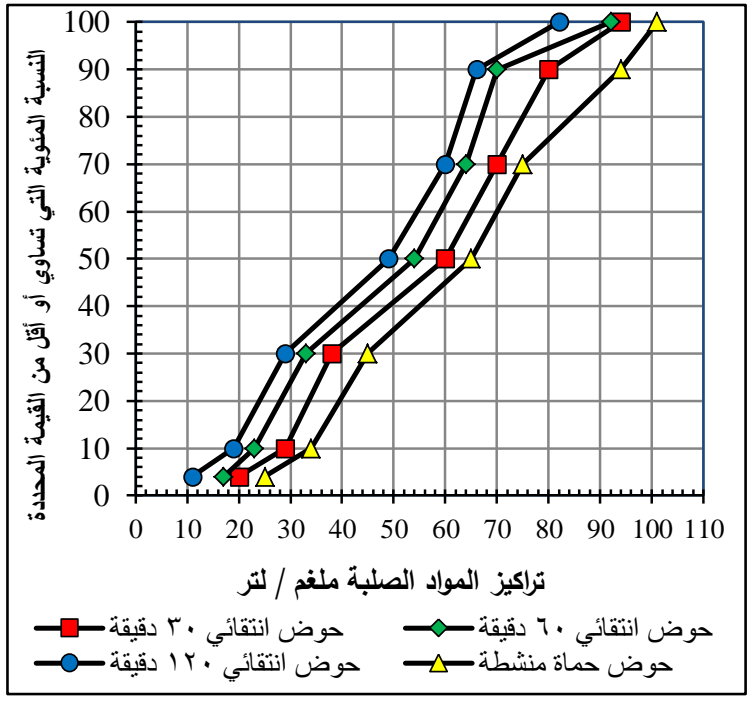

الثكل (5): التوزيع التكراري التراكمي لتراكيز المواد الصلبة العالقة الخارجة من الوحدات الاختبارية للمرحلة التثغيلية الثانية (وقت التعويق = 24 ساعة التبة

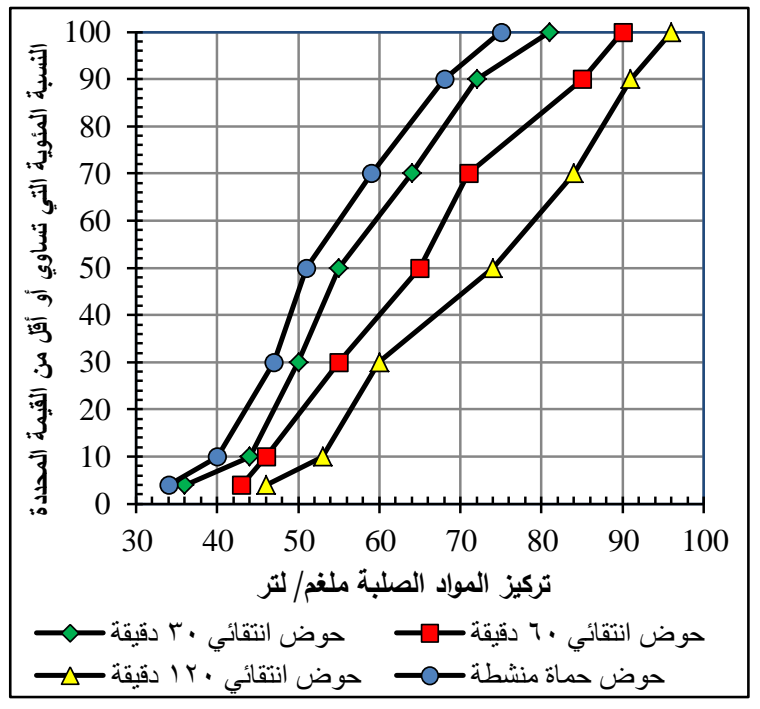

الثكل (4): التوزيع التكراري التراكمي لتراكيز المواد الصلبة العالقة الخارجة من الوحدات الاختبارية للمرحلة التشغيلية الأولى (وقت التعويق = 32 ساعة) الاعبدانة 
وكما بلاحظ من الثكل (4)، فان 70 \% من القراءات المسجلة

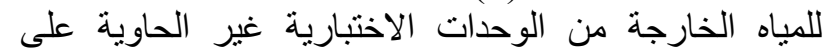

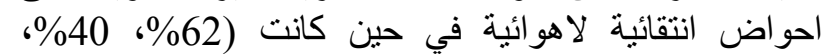
333\% من القراءات المسجلة للوحدات الاختبارية الحاوية

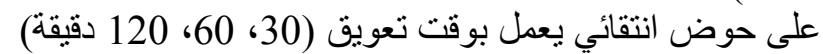

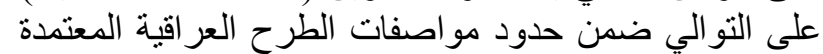

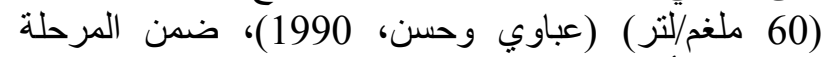

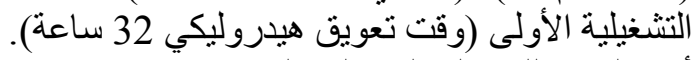

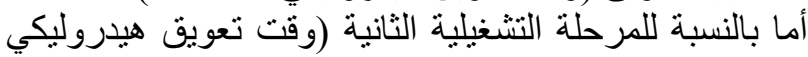

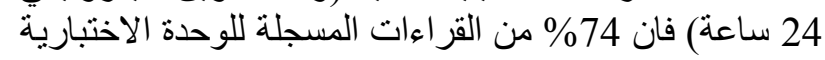

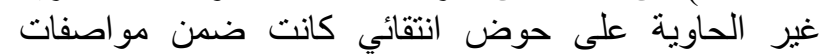

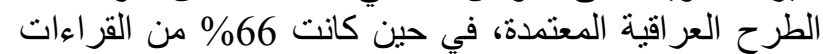

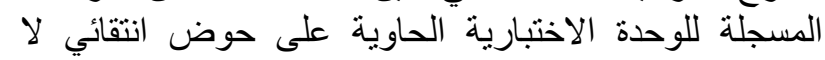

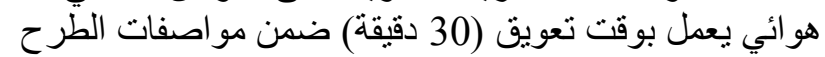

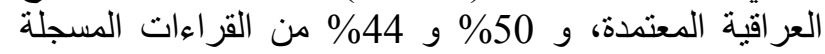

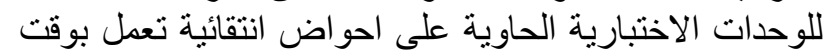

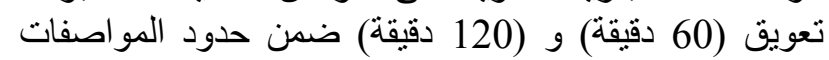

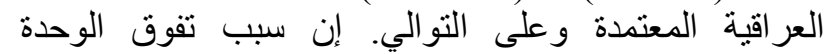

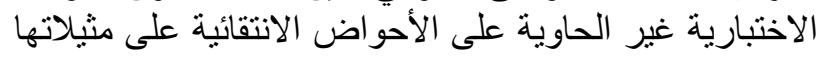

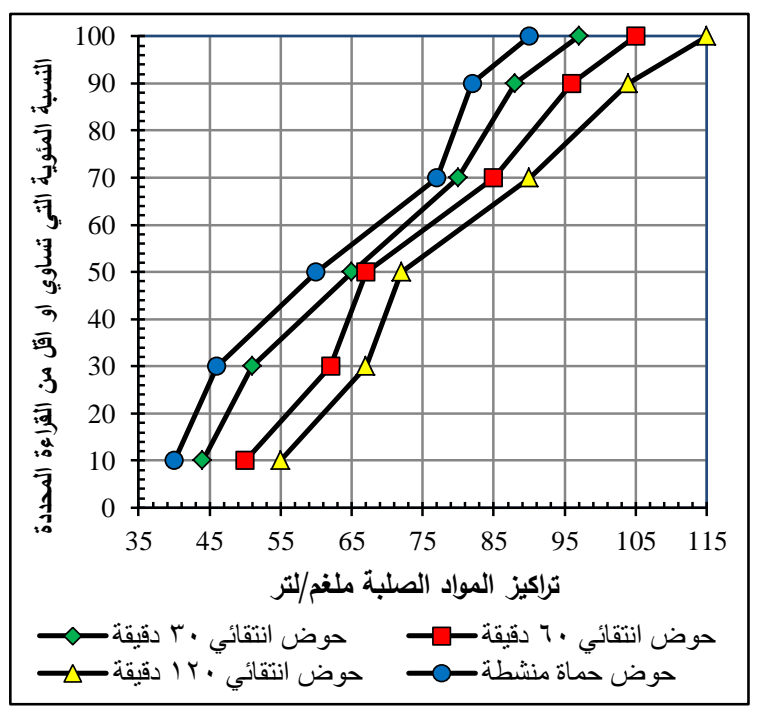

الثكل (6): التوزيع التكراري التراكمي لتراكيز المواد الصلبة العالقة الخارجة من الوحدات الاختبارية للمرحلة الثالثة الثراكية

(وقت التعويق = 18 ساعة الاعة)

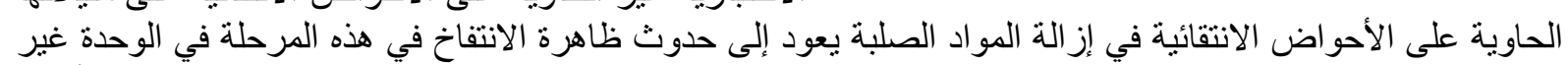

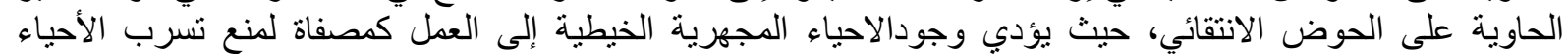

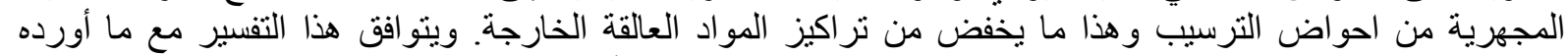

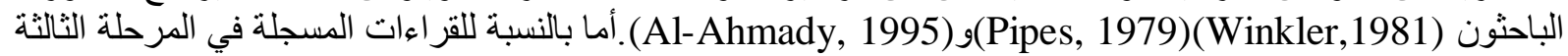

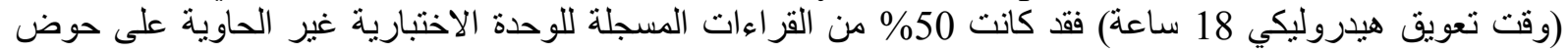

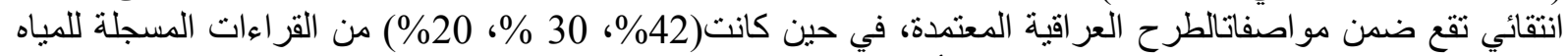

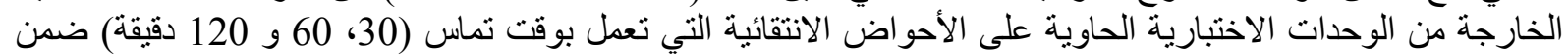
حدودالمو اصفات العر اقية المعتمدة و على التوارئي.

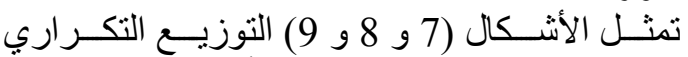

\section{3. تأثير الاحواض الانتقائية اللاهوائية في كفاءة إزالة الفسفور}

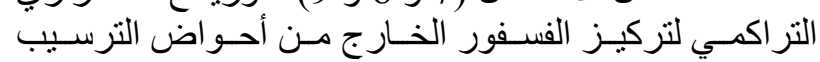

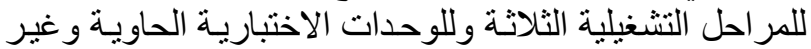
الحاوية على الأحو اض الانتقائية اللاهو ائية.

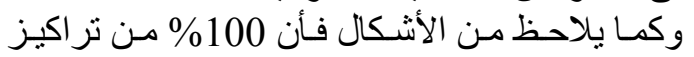

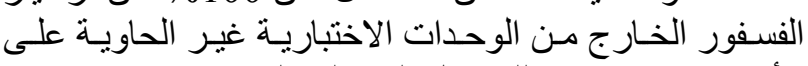

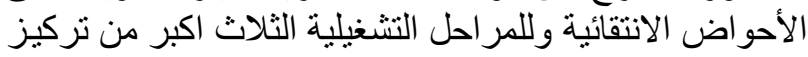

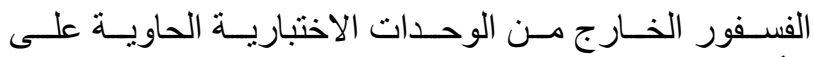
الأحو اض الانتقائية ولجميع المراحل التشغيلية.

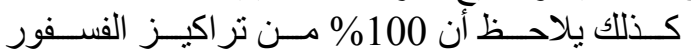

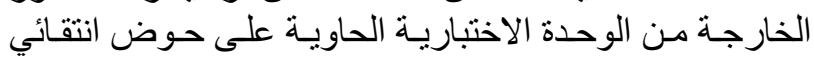

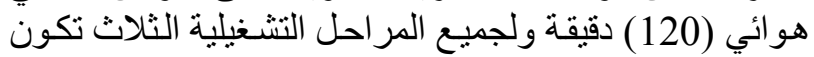

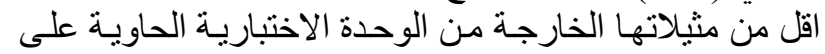

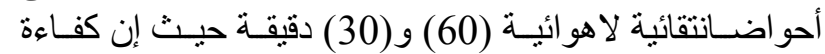

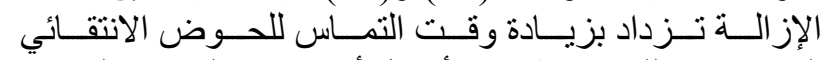

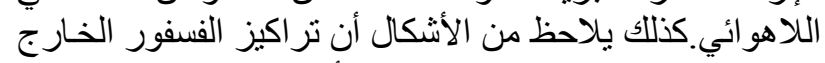
من الوحدات الاختبارية الحاوية على أحواض اضنت انتقائية لا هو ائية

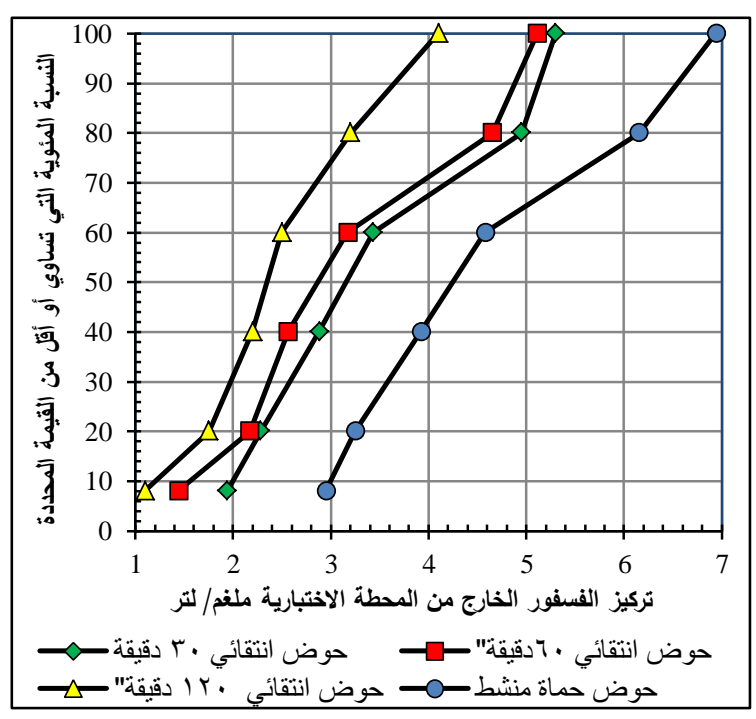

الثكل (7): التوزيع التكراري التراكمي لتراكيز الفففور من

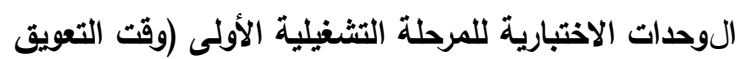


تقل بانخفاض وقت التعويق الهيدروليكي للوحدة الاختبارية وحسب المرحلة التشغيلية.

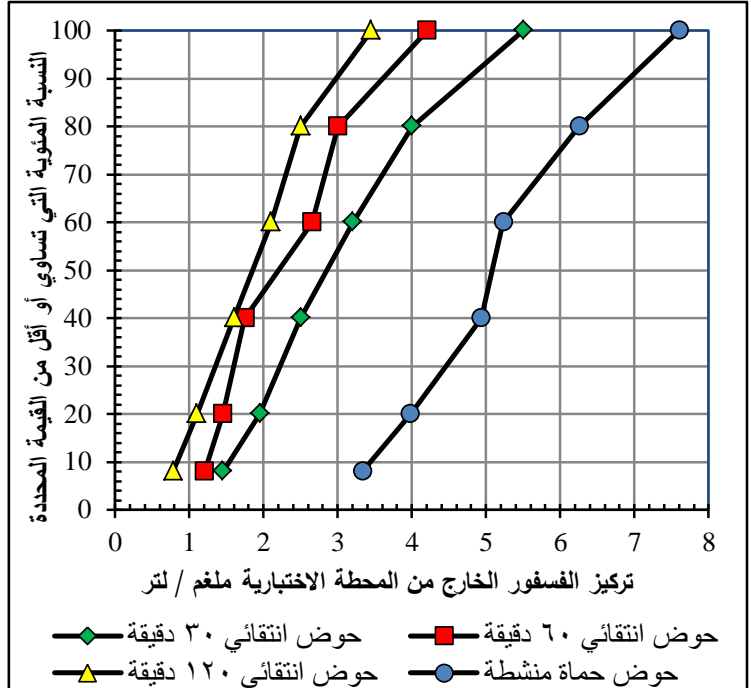

الثكل (9): توزيع التكراري التراكمي لتراكيز الفسفور

الخارجة من الوحدات الاختبارية للمرحلة التشغيلية الثالثة

$$
\text { (وقت التعويق = } 18 \text { ساعة) }
$$

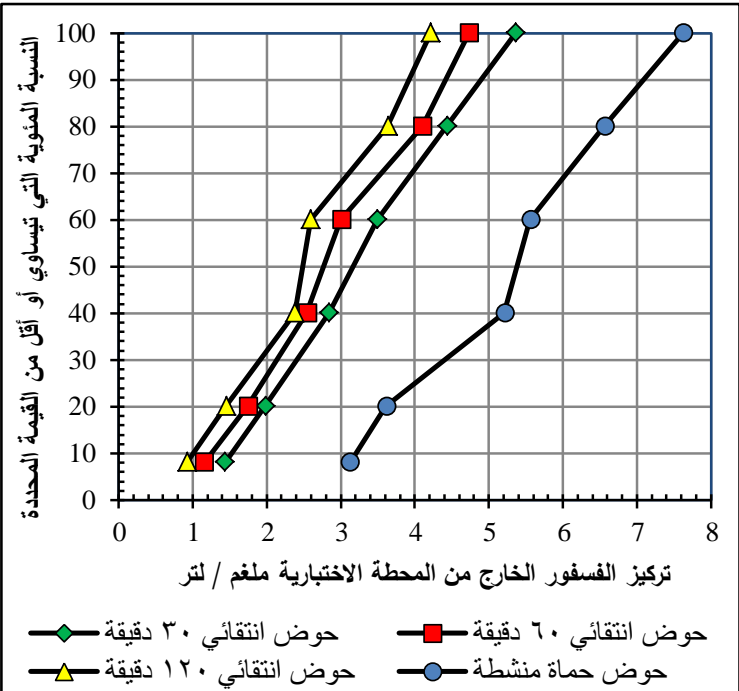

الثكل (8): التوزيع التكراري التراكمي لتراكيز الفسفور من

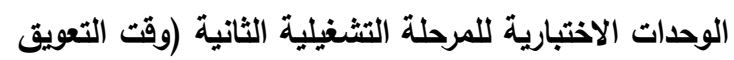

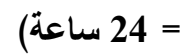

4.تأثير وقت التعويق الهيدروليكي للمفاعل البيولوجي الهوائي في كفاءة إزالة المواد العضوية

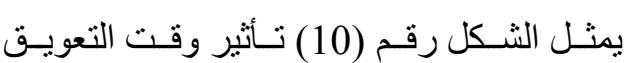

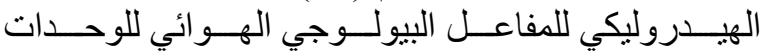

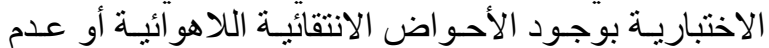

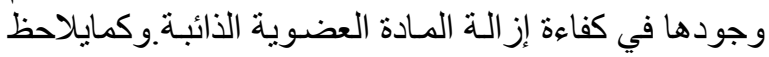

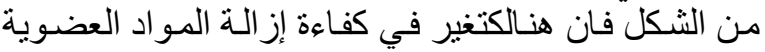

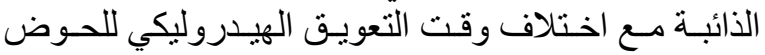

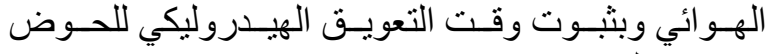

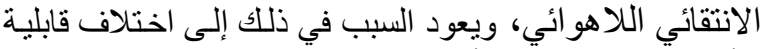

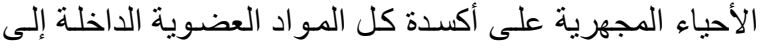

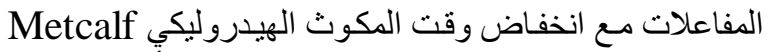
كذلك يلاحظ من الثكل أن الوحدات (and Eddy, 2003)

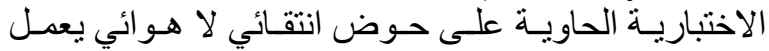
بوقت تعويق هيدروليكي (120) دقيقة أكثر كفاءة بإزاز الـة الته

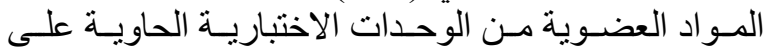

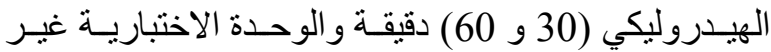

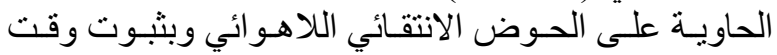

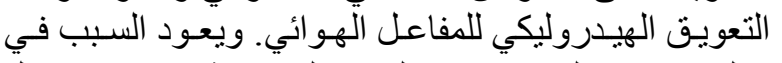

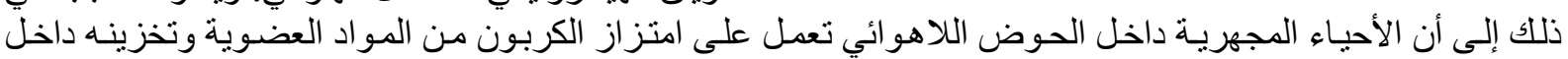

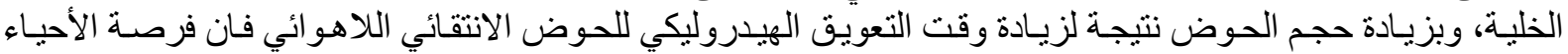

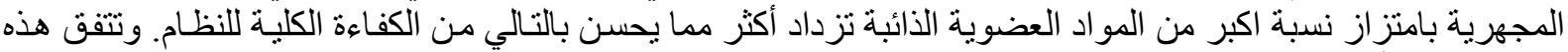
النتيجة مع ما أورده الباحثون Chudob (and Wanner, 1989) و (Daigger and Nicholson, 1990). 


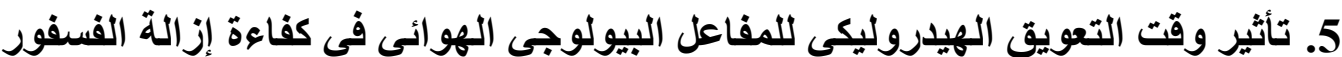

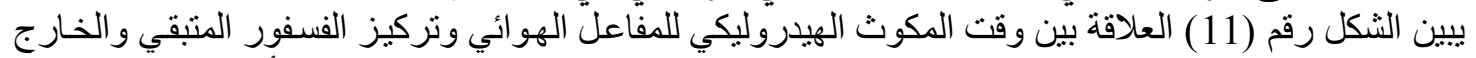

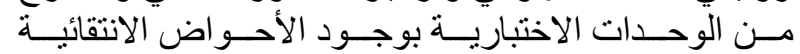

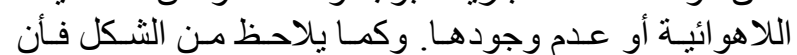

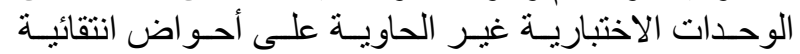

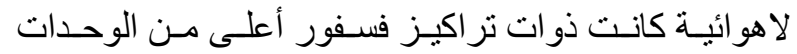

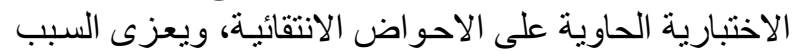

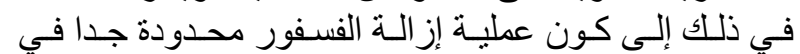

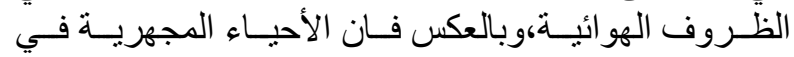

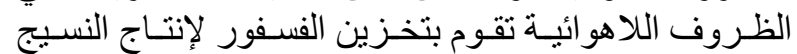

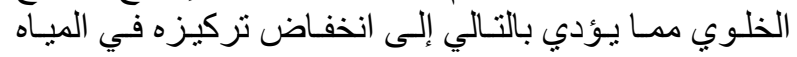

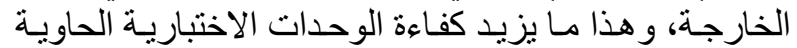

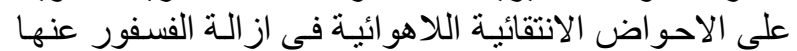

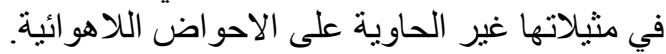

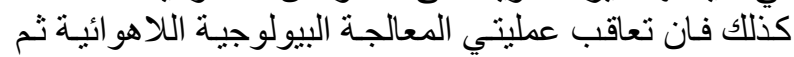

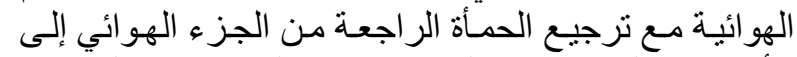

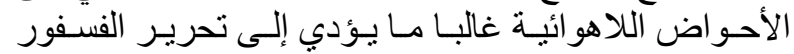

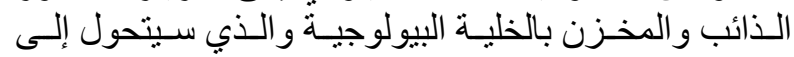

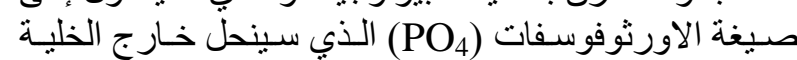

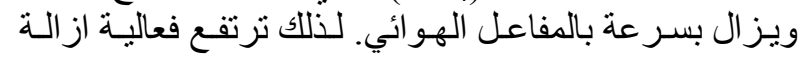

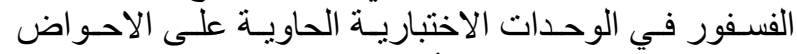

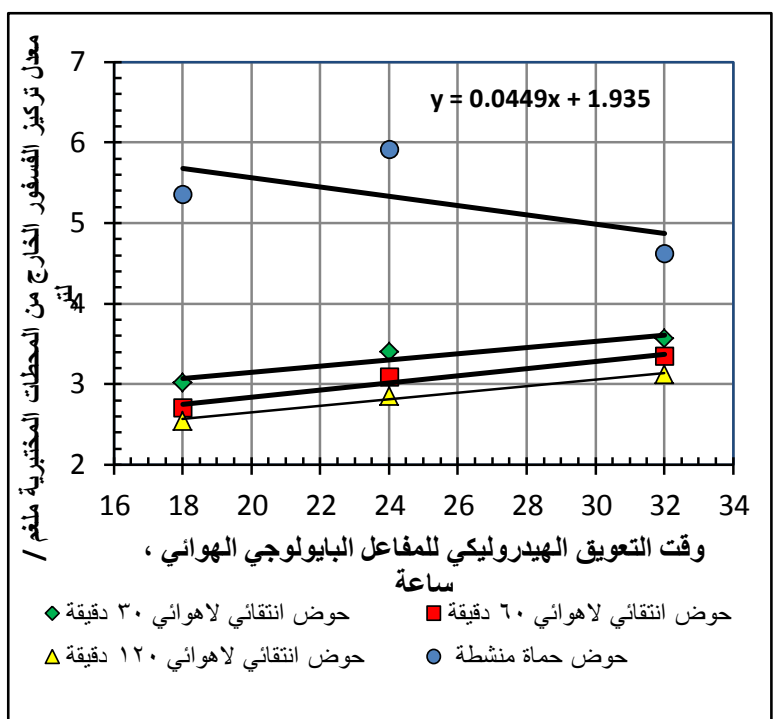

الثكل (11): العلاقة بين وقت المكوث الهيدروليكي للمفاعل

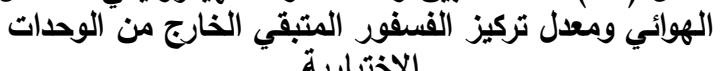

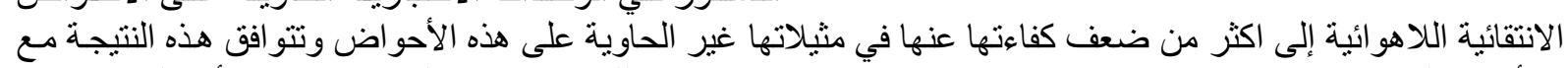

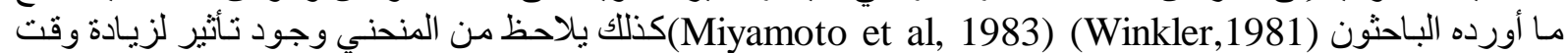

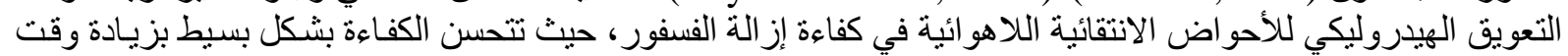

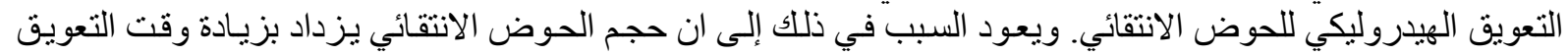

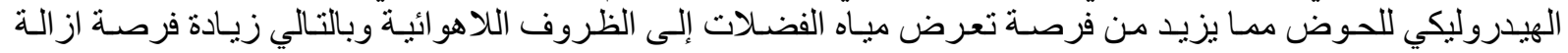

\section{5. تأثير نسبة حجم الحوض الانتقائي اللاهوائي إلى حجم المفاعل الهوائي في كفـاءة إزالة المـواد}

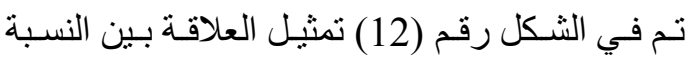

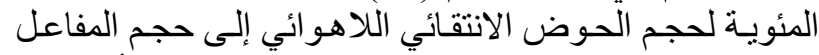

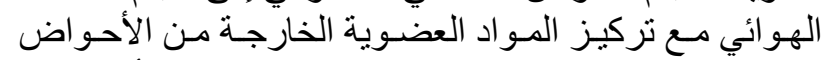

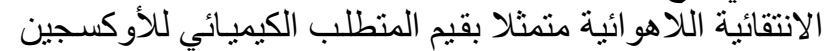

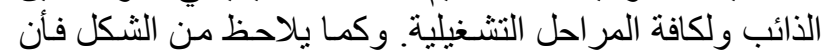

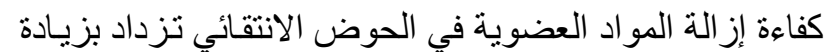

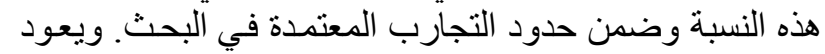

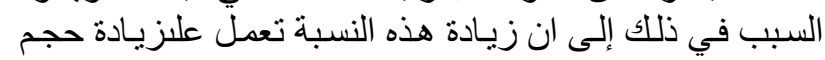

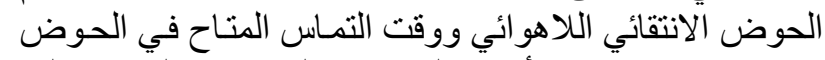

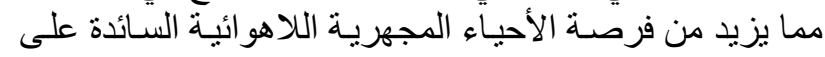

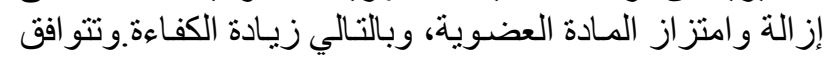

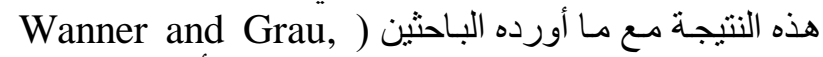

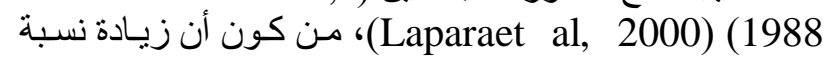

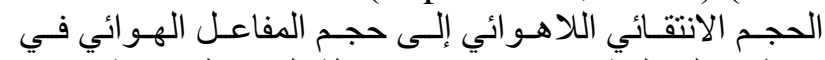

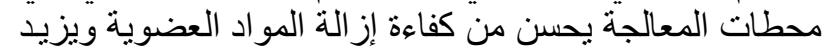

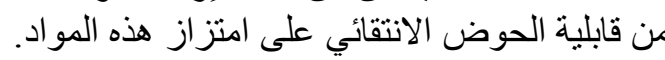

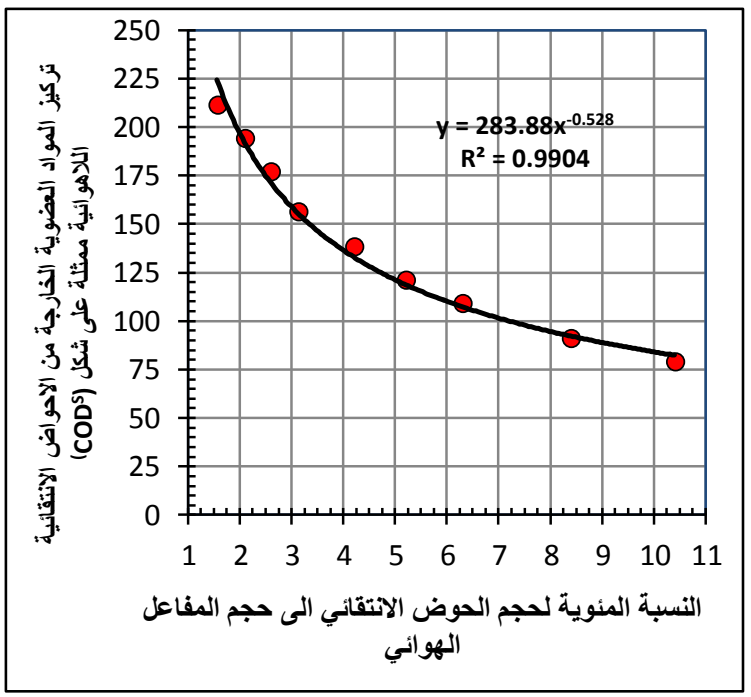

الثكل (12): العلاقة بين النسبة المئوية لحجم الحوض المولئ

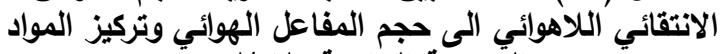
العضوية الخارجة،ملفم/لتر. 
6. تأثير نسبة حجم الحوض الانتقائي اللاهوائي إلى حجم المفاعل الهوائي في معامـل دليل الحمـأة الحجمي (SVI)

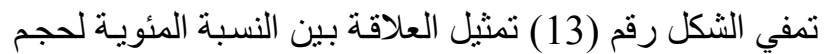

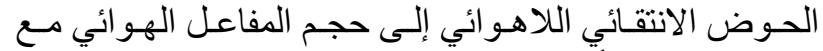

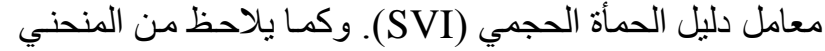

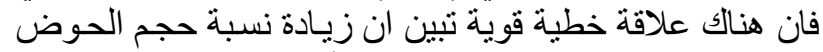

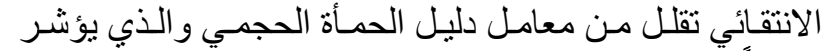

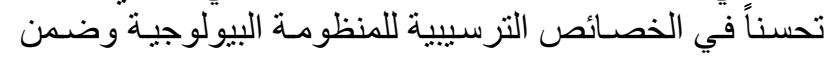

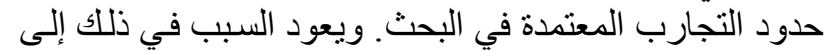

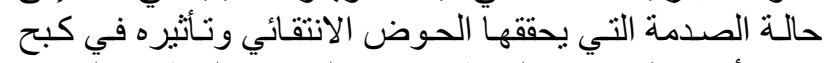
نمو الأحياء المجهرية الخيطية ذوات المساحةّة السطحية الَّبيرة و الصسبة الترسب في المنظومـة وباتجاه تغليب سيادة الأحياء

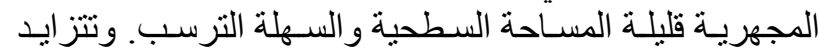

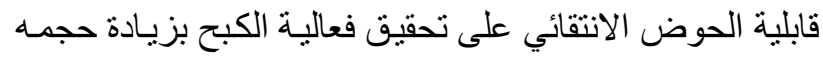

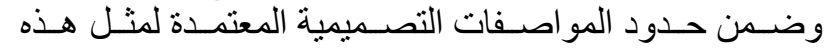

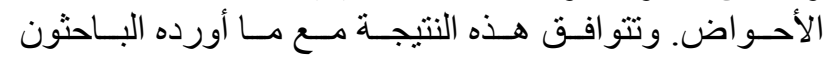
Salameh and Malina, ) و (Sezginet al, 1980) 1989) حيث أوصوا بان تكون نسبة حجم الحوض الانتقائي

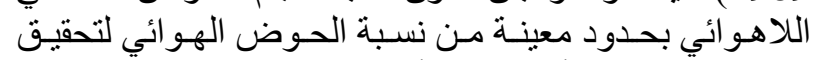
خصائص ترسيبية أفضل للحمأة

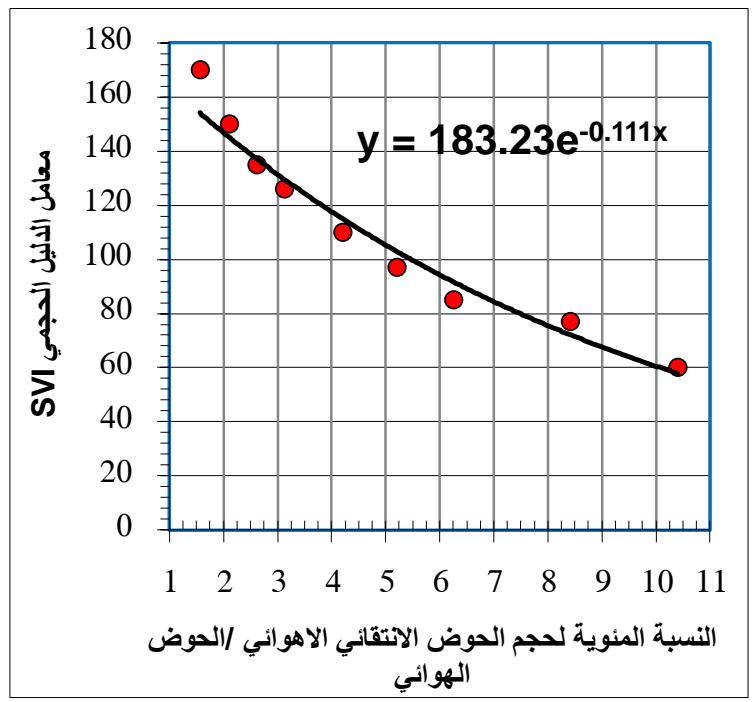

الثكل (13): العلاقة بين النسبة المئوية لحجم الحوض الكبل

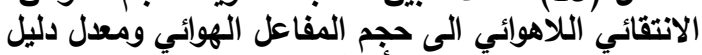
الحمأة الحجمي المجل

7. تأثير نسبة حجم الحوض الانتقائي اللاهوائي إلى حجم المفاعل الهوائي في كفاءة ازالة الفسفور

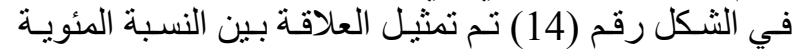

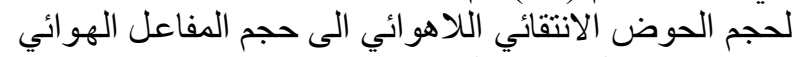

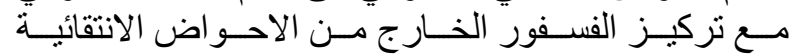

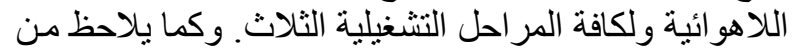

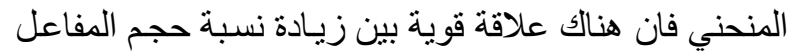

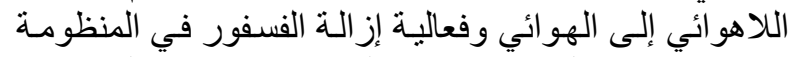

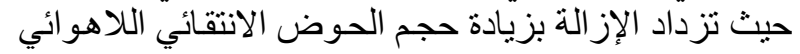

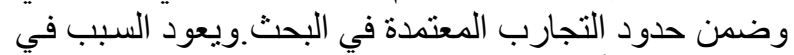

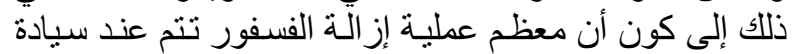

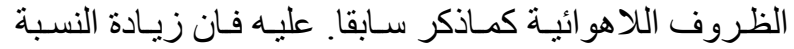

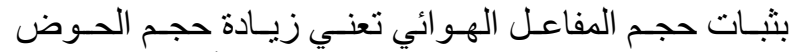

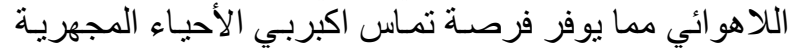

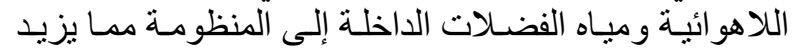

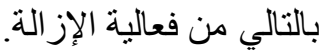

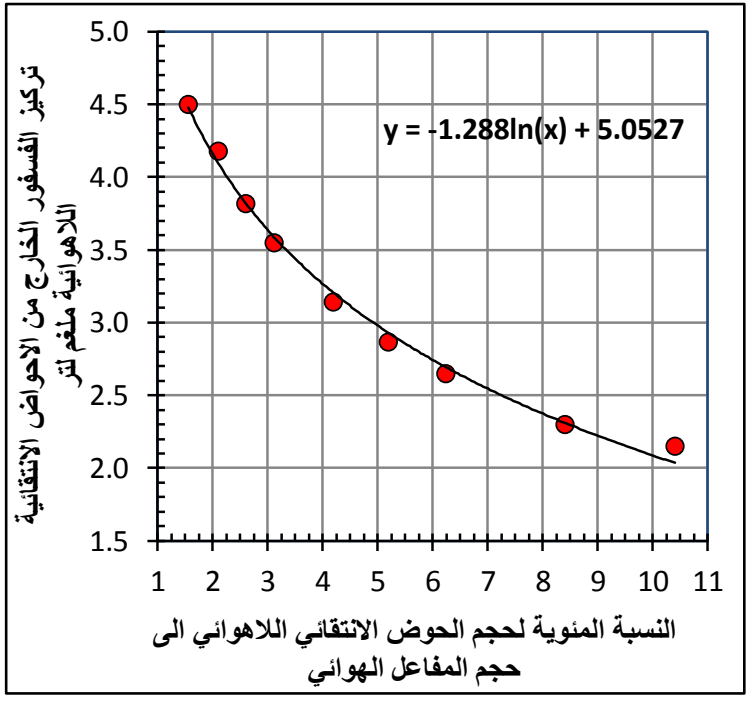

الثكل (14): العلاقة بين النسبة المئويـة لحجم الحـوض

الانتقـائي اللاهـوائي الـى حجم المفاعل الهـوائي وتركيز الفسفور الخارج،ملغم/لتر. 
الاستتناجات

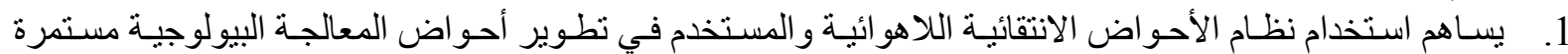

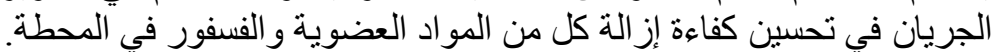

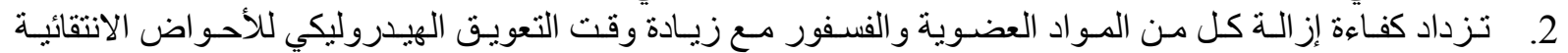

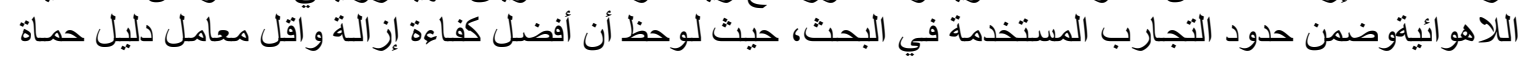

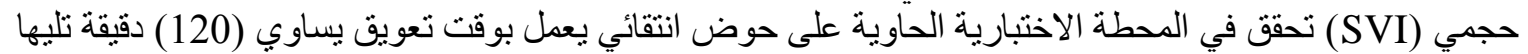

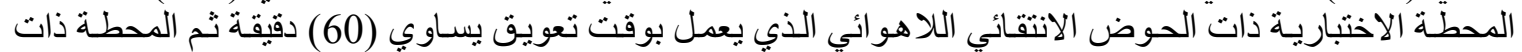

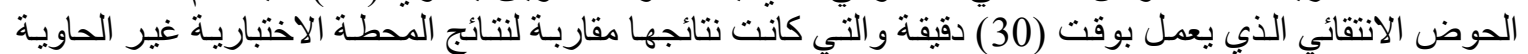
على الأحو اض الاضنتقائية.

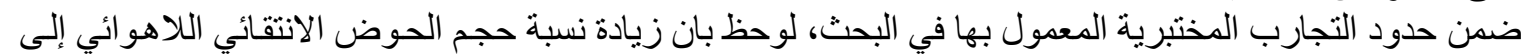

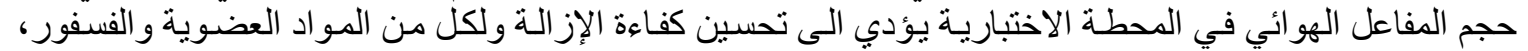
وتحسين قيم معامل دليل الحماة الحجمي للمحطة العارئ الاختبارية.

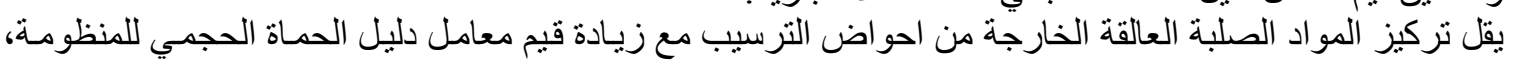

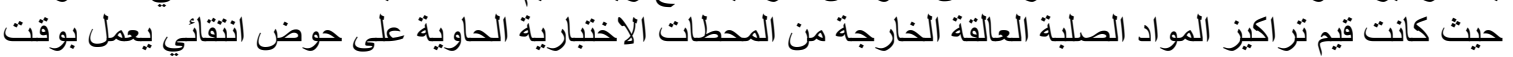

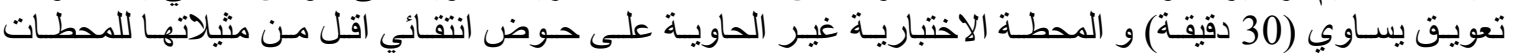

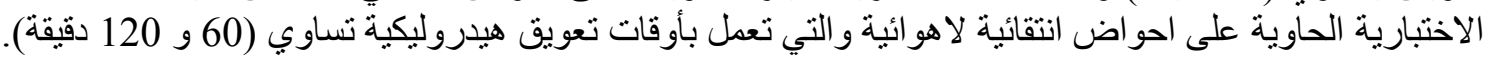

التوصيات 1. دراسة تأثير الصدمات السمية الموجودة في مياه الفضلات المطروحهة من المستشفيات أو الصناعات المختلفة على سلوك المعالجة البيولوجية بوجود الأحواض اضئ الانتقائية اللاهو ائية.

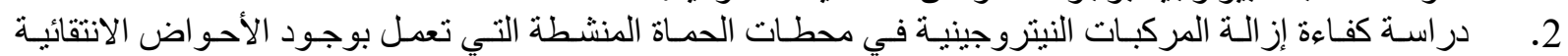

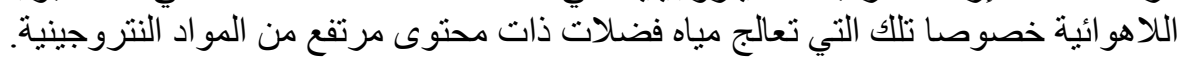

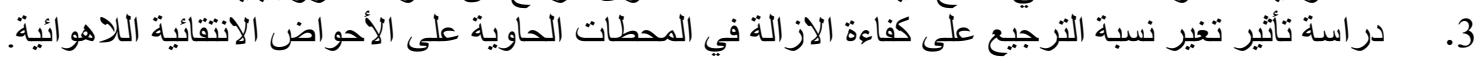

\section{المصادر}

Al-Ahmady, K. K., (1995), “Oxidation ditches with interchannel clarifier process to treat industrial wastes," M.Sc. thesis, University of Mosul

Albertson, O.E. and Hendricks, P. (1991),"Bulking and Foaming Organism Control at Phoenix, Arizona WWTP”, Water Sci. Technol., 26:3-4, 461

Andrea Jobbagy, FerencFarkas, GY. Garai, BelaSevella. and T. Oszoly(2001), "Trial operation of selector at the northpest wastewater treatment plant,"periodicaPolytechnica SER. Chem. ENG. Vol.45, NO.1, PP.41- 52.

Aurola, A. ; Beun, J. J.; Copp, J.; Morgenroth, E.; Loosdrecht, M. C. M. ; and Winkler, S. (2000), "Unbalanced growth", European Cooperation in the field of Scientific and Technical Research Conference, 6-7 April 2000, Delft The Netherlands.

Banoub, A. (1982), "Reducing Energy Consumption: How Two Communities Did It", Water Pollut. Control Fed. Highlights, 4, 11.

Chudoba, J. and Wanner, J. (1989), “Notes on Oxic, Anoxic, and Anaerobic Selectors”, Newsletter Spec. Group Activated Sludge Population Dyn., 1:2, 46

Daigger, G. T. and Nicholson, G. A. (1990), “ Performance of Four Full-Scale Nitrifying Wastewater Treatment Plants Incorporating Selectors”, Res. J. Water Pollut. Control Fed., 62, 676.

Harper, W. F. and Jenkins, D. (2001),"The Effect of an Initial Anaerobic Zone on the Nutrient Requirements of Activated Sludge", Proc. WaterEnviron Fed., WEFTEC 2001, on disc 
Jayh H. Niec and Daniel K Cha (2000), “Influence of Anoxic Selectors on Heavy Metal Removal by activated sludge ",Bioresource Bioprocess Eny 2000. Department of Civil and Environmental Engineering, University of Delaware.

Lapara T.M., Konopka A., Nakatsu C.H. and Alleman E., (2000), “Thermophilic aerobic wastewater treatment in continuous flow bioreactor", Journal of environmental engineering, Vol. (126), No. (8).

Mangrum, C. R. l. (1998), "The effect of anoxic selectors on the control of activated sludge bulking and foaming", M.Sc. Thesis, Virginia Polytechnic Institute and state University

Metcalf \& Eddy, Ins (2003), "Wastewater engineering treatment disposal McGraw-Hill, Inc, New York.and reuse", Fourth Edition .

Micahael Richard, Ph. D (2003), "Activated sludge microbiology problems and their control ”,Annual USEPA National Operater Conference, Buffalo, NY, June 8,2003

Miyamoto-Mills, J., Larson, J., Jenkins, D., and Owen, W. F. (1983), "Design and Operation of a Pilot-Scale Biological Phosphate Removal Plant at Central Contra Costa Sanitary District", CA, Water Sci. Technol., 15:3-4, 153

Nayef Z. AL-Mutairi (2008), “Aerobic selectors in slaughterhouse activated slugesystem " Available on line 15 July .

Peng, Y.; Gao, C.; Wang, S.; Ozaki, M. and Takigawa, A. (2003), "Non-filamentous sludge bulking caused by a deficiency of nitrogen in industrial wastewater treatment", Water Science and Technology, Vol. 47, No. 11 pp. 289-295

Pipes, W. O. (1979), "Bulking, deflocculation and pinpoint floe", J. Water Poll. Control Fed. 51(1): 62-70.

Ramalho, R. S. (1977) “Introduction to wastewater treatment process", Academic press,Inc.,Canada

Richard J., Walter J., Christian D., Alan F., (2000), “Design and start-up of an advanced treatment system for high strength wastewater from a chemical plant”, PMC Technologies, Pennsylvania

Salameh, M. F. and Malina, , J. F. Jr (1989), "The effects of sludge age and selector configuration on the control of filamentous bulking in the activated sludge process", Journal of Water Pollution Control Federation.Vol.61,NO 9,PP 1510-1522.

Sezgin, M., Palm, J. H., and Jenkins, D. (1980), “The Role of Filamentous Microorganisms in Activated Sludge Settling”, Water Technol., 12:6, 171.

Shao, Y. J. and Jenkins, D. (1989), “ The Use of Anoxic Selectors for the Control of Low F/M Activated Sludge Bulking”, Water Sci. Technol., 21:11, 609.

Tomlinson, E. J. (1978), “The Effect of Anoxic Mixing Zones upon the Settleability of Activated Sludge, unpublished report", Water Research Centre, Stevenage, U.K..

Wanner, J. and Grau, P. (1988), "Filamentous Bulking in Nutrient Removal Activated Sludge Systems", Water Sci. Technol., 20:4-5,Lapara T.M., Konopka A., Nakatsu C.H. and Alleman E., (2000), "Thermophilic aerobic wastewater treatment in continuous flow bioreactor", Journal of environmental engineering, Vol. (126), No. (8).

Winkler, M. A. (1981), “Biological Treatment of Wastewater”, 1st ed., Ellis Horwood Ltd. Publishers; England.

$$
\text { تم اجراء البحث في كلية ألهندة = جامعة ألموصل }
$$

